\title{
Effect of genetic and patient factors on warfarin \\ pharmacodynamics following warfarin withdrawal: implications for patients undergoing surgery
}

Running title: INR decline after warfarin withdrawal

Salah Abohelaika ${ }^{1}$, Hilary Wynne ${ }^{2}$, Peter Avery $^{3}$, Emmanouela Kampouraki ${ }^{1}$, Farhad Kamali ${ }^{1}$

1. Institute of Cellular Medicine, Newcastle University and Newcastle upon Tyne Hospitals, NHS Foundation Trust, Newcastle upon Tyne, NE1 7RU, UK

2. Department of Older People's Medicine, Newcastle upon Tyne Hospitals, NHS Foundation Trust, Freeman Hospital, Freeman Road, Newcastle upon Tyne, NE7 7DN, UK

3. School of Mathematics and Statistics, Newcastle University, Newcastle upon Tyne NE1 7RU, UK

Text word count: 2877 (excluding abstract, tables and references)

Abstract word count: 205

No of tables: 2

No of figures: 0

Corresponding Author: Professor Farhad Kamali, Institute of Cellular Medicine, Newcastle University, and Newcastle upon Tyne Hospitals, NHS Foundation Trust, Newcastle upon Tyne, UK. Fax: +44 1912225827 Email address: (farhad.kamali@ncl.ac.uk). Tel no: +44 1912088043

Keywords: Warfarin, anticoagulation, thromboembolism, CYP2C9, VKORC1, surgery 


\section{Abstract}

Introduction

Warfarin therapy is stopped for a fixed period prior to surgery to minimise risk of perioperative bleeding. However, anticoagulation subsides at varying rates among different patients. We evaluated the influence of genetic (CYP2C9 and VKORC1), patient and clinical factors on warfarin clearance and the decline in INR following warfarin withdrawal.

\section{Materials and methods}

131 patients completing a course of warfarin provided blood samples over 9 days for initial genotyping, and measurement of INR and plasma warfarin enantiomer concentrations.

\section{Results}

S-warfarin clearance was significantly lower in patients with either CYP2C9 single ( ${ }^{* 2}$ or *3) or double $\left({ }^{*} 2{ }^{*}\right.$ or $\left.{ }^{*} 2{ }^{*}\right)$ variant alleles compared to those with wild-type genotype $(P<0.001)$. Regression analysis revealed that patient age $(P=0.037)$ and CYP2C9 *2*2 \& *2*3 genotype $(\mathrm{P}=0.005)$, but not VKORC1 genotype, significantly affected the time taken for the resumption of normal coagulation (INR value declining to $\leq 1.5$ ).

\section{Conclusions}

The inter-individual variability in the time needed for normal coagulation to resume following warfarin withdrawal is influenced, in the main, by variance in S-warfarin clearance, which in turn is affected by CYP2C9 polymorphism and age. Cost-effectiveness of pharmacogenetics-based algorithms incorporating CYP2C9 genotype and patient age could be increased if used not only to guide dosing decisions but also estimation of the correct length of time needed for individual patients to stop taking warfarin prior to surgery. 


\section{Introduction}

Warfarin is prescribed as a 50:50 racemic mixture of $(R)$ - and (S)- warfarin enantiomers, with the latter being 3-5 times pharmacologically more potent than the former. (S)-warfarin hydroxylation is influenced by polymorphisms in the CYP2C9 gene; CYP2C9*2 and CYP2C9*3 variants encoding enzymes that are respectively around $12 \%$ and $5 \%$ as efficient as the wild-type (CYP2C9 * $\left.1{ }^{*} 1\right)$ enzyme $(1-3)$.

Vitamin K epoxide reductase, the pharmacological target enzyme for warfarin, recycles vitamin $\mathrm{K}$ epoxide to the reduced active form of vitamin $\mathrm{K}$ (vitamin $\mathrm{K}$ hydroquinone), an important cofactor needed in the functionalisation of clotting factors II, VII, IX, and X by $\gamma-$ glutamyl carboxylation of their $\mathrm{N}$-terminals. The enzyme is encoded by the vitamin $\mathrm{K}$ epoxide reductase complex subunit 1 gene (VKORC1); polymorphisms in this gene have been identified and linked with clotting factor deficiencies and warfarin resistance (4) .

Although the contribution of CYP2C9 and VKORC1 genotypes to warfarin dose requirements is well established (5-7), the extent of their influence upon the recognised inter individual variation in the rate of INR decay after cessation of warfarin therapy in patients awaiting surgery is not. An earlier study indicated that sensitivity to warfarin, advanced age and extreme elevation of the INR are independent risk factors for prolonged delay in return of a supra-therapeutic INR to the target range (8). In patients following established recommendations to stop their warfarin 5 days prior to an invasive procedure (9), rate of INR decline varies, with $23 \%$ having an INR $>1.2$ after 4.7 days of withdrawal from warfarin in one study (10), $7 \%$ having a pre-operative INR $>1.5$ after five days with subsequent need for vitamin $\mathrm{K}$ administration prior to surgery in another (11). In our previous study of the effect of CYP2C9 genotype upon INR decline, patients with two CYP2C9 variant alleles $\left(\right.$ CYP2C $9^{*} 2^{*} 2$ or CYP2C9*2*3) were found to be over 8 times more likely $(95 \%(12)=2.25$ 33.25) to have an INR of $\geq 1.5$ on the day before planned surgery, compared to those with either ${ }^{*}{ }^{*} 1,{ }^{*} 1{ }^{*} 2$ or ${ }^{*} 1{ }^{*} 3$ genotypes. The possible impact of $V K O R C 1$ polymorphism on the 
fall in INR was not evaluated (13). The aim of the current study was to characterise further the contribution of genetic, patient and clinical factors toward warfarin elimination and its changing pharmacodynamics in patients with thromboembolic disease following warfarin withdrawal and to determine the potential clinical utility of a personalized pharmacogeneticsbased approach in guiding management of patients on warfarin scheduled for invasive surgery.

\section{Methods}

The study had the approval of the Newcastle upon Tyne Ethics Committee. All patients provided written informed consent according to the Declaration of Helsinki. One hundred and thirty five Caucasian patients aged over 18 years, completing a course of warfarin for indications of either venous thromboembolism (VTE), or atrial fibrillation (warfarin therapy stopped after successful cardioversion), with a target INR of 2.0-3.0 and stable control of anticoagulation (defined as having a stable warfarin dose for at least the previous 2 clinic visits a minimum of one week apart), were identified through the anticoagulant clinic records at the Newcastle upon Tyne Hospitals NHS Foundation Trust, UK. Any patient with a condition which might affect warfarin metabolism, notably congestive cardiac failure, hepatobiliary or renal disease or active cancer, taking medication known to interact with warfarin, or drinking more than the UK recommended safe limits (14 units per week for men and women) (14) was excluded.

On the day of warfarin withdrawal, demographic data of age, weight, sex, and indication for anticoagulation therapy, medical diagnoses, daily warfarin dose, concomitant medication and alcohol intake were recorded. A blood sample $(10 \mathrm{ml})$ was taken for measurement of baseline INR and CYP2C9 and VKORC1 genotyping. Thereafter, each patient attended the clinic on 3-4 separate occasions, spread over 9 days, beginning the day after their last dose of warfarin, with some variation to avoid scheduling conflicts (e.g. weekends) and missed appointments. A venous blood sample $(10 \mathrm{ml})$ was collected at visit one (16-20 hours after the last dose of warfarin) for measurement of steady-state plasma warfarin enantiomer 
concentrations and INR. A venous blood sample $(10 \mathrm{ml})$ was taken at each of the remaining study visits for INR measurement only.

Plasma warfarin enantiomer concentrations were determined by high performance liquid chromatography (HPLC) using the method of Naidong, et al (15). The limit of detection of the extracted samples was $2 \mathrm{ng} / \mathrm{ml}($ signal $/$ noise ratio $=3$ ). The inter-day coefficient of variation for S- and R- warfarin at $690 \mathrm{ng} / \mathrm{ml}$ was $4.2 \%$ and $3.8 \%$, respectively. CYP2C9 and VKORC1 -1639 genotyping were carried out according to an established method (5). The rate of decline in INR for individual patients was determined from the slope of the plot of INR versus time. S- and R- warfarin clearance $(\mathrm{CL})$ was calculated according to the formula: $\mathrm{D} /(2 \mathrm{t} X$ Css.av), where $\mathrm{D}$ is the daily dose of warfarin $(\mathrm{mg}), \mathrm{t}$ is the dosing interval (24 hours), and Css.av is the average steady-state plasma warfarin enantiomer concentration (mg/L), assuming that warfarin compliance and bioavailability are 100\% (16).

\section{Sample size calculation}

According to previous studies INR reached a value of $<1.5$ in $74 \pm 19$ hours following warfarin withdrawal $(10,11)$. To achieve $80 \%$ power and to detect a 15 hour difference in the time needed to reach an INR $<1.5$ between individuals with the CYP2C9 * $1{ }^{*} 1$ genotype and either those with at least one variant allele (i.e. either $C Y P 2 C 9^{*} 2$ or $C Y P 2 C{ }^{*} 3$ ) and allowing for the expected proportion in the three genotype groups, a sample size of 135 patients were needed to be studied.

\section{Statistical analysis}

Statistical analysis of the data was carried out using Minitab software version 17 (Coventry, UK). The effects of study variables on INR were assessed using multiple and logistic regression models. By regressing the logarithm of INR against time for each individual, the 
time for INR to reach 1.5 was estimated. This estimated time was modelled using CYP2C9 and VKORC1 genotypes, and other study variables. When necessary, data were transformed into their logarithmic values to approach normality. One-way analysis of variance (ANOVA) was used for comparison of continuous data. $A \mathrm{P}<0.05$ was taken as being statistically significant. Data are presented as mean $\pm S D$ unless otherwise stated.

\section{Results}

One hundred and thirty five patients were recruited into the study. However, for statistical analysis data were available for 131 participants [73 (56\%) males] as four later withdrew from the study due to inability to attend study visits. The age range for the study cohort was 21-92 years; there was no significant difference in mean age between males and females (62 \pm 15 versus $64 \pm 18$ years). At the point of recruitment, patients were taking a median of 2 drugs (IQR 1-5), excluding warfarin, 1 of which affected the cardiovascular system. Of drugs reported as potential inhibitors of CYP2C9, 1 patient was taking clopidogrel and 1 phenytoin, a drug which is also reported as a potential inducer of CYP2C9. Two patients were taking the potential inducer, carbamazepine. Forty one patients were taking statins of whom 30 were taking simvastatin, 9 atorvastatin, 1 rosuvastatin and 1 pravastatin. No patient was taking amiodarone or fluvastatin. As well as indications for warfarin (atrial fibrillation $11 \%$, deep venous thrombosis $61 \%$, pulmonary embolism $26 \%$, and valvular prosthesis $2 \%$ ), the median number of comorbidities was 1 (IQR 1-2), most commonly cardiovascular disease (69\%). The frequency of CYP2C9 and VKORC1 genotypes in the study population are presented in Table 1.

On the screening day following the final warfarin dose (warfarin dose usually taken early evening the day before), mean INR was $2.4 \pm 0.6$. At the first study visit (16-20 hours after the last warfarin dose), the proportion of INR values $\geq 1.5$ among those with $C Y P 2 C 9$ wild-type, single variant, and double variant genotype was $78 \%, 94 \%$, and $100 \%$, respectively; 
CYP2C9 genotype was the only predictor of an INR $<1.5(P=0.009)$ at the first visit; the odds ratio for the single variant genotype relative to the wild-type was $0.22(95 \% \mathrm{Cl}=0.06,0.79)$. The proportion of INR values $\geq 1.5$ at the second visit (mean of 102 hours) among those with CYP2C9 wild-type, single- and double-variant alleles was 9\%, 15\%, and 40\%, respectively, but these differences were no longer statistically significant; no further genetic influences were identifiable beyond the second visit as most of the INR values were close to 1.0 by the third and fourth study visits.

According to regression analysis, which included the variables of VKORC1 and CYP2C9 genotype, age, weight, sex, warfarin dose, comorbidities, concurrent drugs, and alcohol, CYP2C9 genotype was the only factor which influenced the rate of INR decline ( $P=0.014)$; the slope of the regression lines for INR for CYP2C9 (single and double variants) were significantly shallower than that for CYP2C9 wild-type. Regression analysis revealed that the time required to fall to an INR value of 1.5 in patients initially within their $2-3$ target range was significantly associated with both patient age $(\mathrm{P}=0.037)$ and CYP2C9 genotype $(P=0.005)\left(R^{2}=15.7 \%\right)$. Using the regression equation, it can be estimated that for example, a 90-year old will take 18 hours longer to reach an INR of 1.5 than a 30-year old, and, for example, at the age of 60 , in patients with either ${ }^{*} 1^{*} 1,{ }^{*} 1{ }^{*} 2,{ }^{*} 1{ }^{*} 3,{ }^{*} 2{ }^{*} 2$, or ${ }^{*} 2{ }^{*} 3$ genotype, on average it will take $61,63,78,86$, and 100 hours, respectively for the INR to fall to 1.5 . In patients possessing either a single or double CYP2C9 variant allele, S-warfarin clearance was slower (mean difference of $0.1 \mathrm{~L} /$ hour, for both single and double variant alleles; $\mathrm{P}<0.0001$ ), compared to patients with wild-type genotype. Table 2 shows $\mathrm{S}$ - and $\mathrm{R}$ - warfarin clearance values for the various CYP2C9 genotypes. CYP2C9 genotype had no influence upon R-warfarin clearance.

According to the regression analysis, neither VKORC1 genotype, concurrent therapy, comorbidity, patient weight, nor alcohol consumption influenced either the time taken for INR to reach 1.5 , or S- and $\mathrm{R}$ - warfarin clearance. 
As expected, warfarin weekly mean dose was significantly different between patients according to their CYP2C9 $(\mathrm{p}=0.0003)$ and VKORC1 $(\mathrm{P}<0.0001)$ genotype. Patients with CYP2C9 single- or double-variant alleles had a lower weekly mean dose requirement compared to the wild-type carriers; mean difference of $11 \mathrm{mg}(P=0.001)$ and $15 \mathrm{mg}(P=0.03)$, respectively. Patients with VKORC1 AA or AG genotypes had lower weekly mean dose requirement compared to those with $G G$ genotype; mean difference of $21 \mathrm{mg}(\mathrm{P}<0.0001)$ and $10 \mathrm{mg}(\mathrm{P}=0.003)$, respectively. Similarly, patients with VKORC1 AA genotype had a lower weekly mean dose requirement compared to those with $A G$ genotype; mean difference $11 \mathrm{mg}, \mathrm{P}=0.02$. Also, as expected, warfarin weekly dose requirement was negatively correlated with age $(r=-0.4, P<0.0001)$.

\section{Discussion}

Patients on warfarin are normally required to interrupt therapy for a fixed number of days prior to an invasive procedure. However, the anticoagulant activity of warfarin (as measured by INR) subsides at different rates among different patients. Discontinuation of warfarin too early may predispose the patient to thrombosis, and stopping it too late may result in perioperative bleeding.

The results of this prospective study which investigated genetic and non-genetic influences upon INR decline support our hypothesis that the time required for the resumption of normal coagulation to take place following the cessation of warfarin therapy is largely dependent upon S-warfarin clearance, which in turn is influenced by CYP2C9 polymorphism and patient age (5). Polymorphisms in CYP2C9 (the main enzyme responsible for S-warfarin metabolism) and differences in patient age (which influences liver size and liver blood flow (17)) thus partly explain the previously reported variation in INR decay in patients withdrawing from warfarin prior to surgery $(10,11)$, accounting for $16 \%$ of the variability noted in this study. The influence of CYP2C9 on the fall in INR following warfarin withdrawal 
observed in this study is in keeping with our previous study findings in that normalization of coagulation takes place more slowly in patients with CYP2C9 mutations, with those with two CYP2C9 variants being more than eight times more likely to have an INR of $\geq 1.5$ on the fifth day of warfarin withdrawal (13).

We also assessed the potential influence of VKORC1 polymorphism, based upon the notion that polymorphisms in VKOR, the pharmacological target for warfarin, can affect the fall in warfarin anticoagulant activity following warfarin cessation. However, VKORC1 polymorphism made no significant contribution to the variability in INR decline in our study population. Two previous studies, one in healthy subjects (18), and the other in patients prior to surgery (19), found that neither VKORC1, nor CYP2C9, influenced the normalization of coagulation following warfarin withdrawal. However, neither study was sufficiently powered to detect the influence of either gene on the fall in INR; this was evident from the very small number of patients identified in either study as having variant alleles for VKORC1 and CYP2C9.

In our self-reported Caucasian study population there were few patients who were of CYP2C9 ${ }^{*}{ }^{*} 2^{*}$ genotype, whilst there was only one patient who was of ${ }^{*} 2{ }^{*} 3$ genotype and no patient was of ${ }^{*} 3{ }^{*} 3$ genotype. As such, it was not possible to evaluate the effect of the individual genotypes on S-warfarin clearance and the fall in INR following warfarin withdrawal. Nevertheless, the frequency distribution for CYP2C9 genotype fits in with that of the Caucasian general population in whom only about $2 \%$ are homozygous and $\sim 20 \%$ are heterozygous carriers of the CYP2C9*2 allele, with a significantly smaller proportion being either homozygous or heterozygous carriers of the CYP2C9*3 allele (20). In common with the literature we found that warfarin dose requirement was significantly influenced by CYP2C9 and VKROC1 genotype (5, 12, 21, 22).

As well as S- warfarin, CYP2C9 has other drug substrates, some of which are potential inhibitors and some inducers of the enzyme $(23,24)$. Amiodarone is the most notable inhibitor, with a $22 \%$ reduction in predicted therapeutic dose having been associated with its 
use in one study (25), $29 \%$ in another with a $12 \%$ lower warfarin dose requirement in patients who took simvastatin (26). Use of CYP2C9 inhibitors amiodarone, statins (simvastatin and fluvastatin), sulfamethoxazole, azole antifungals, and CYP2C9 inducers rifampicin, phenytoin and carbamazepine have also been reported by other studies $(12,25)$ to affect warfarin dose requirement. Of these drugs, only simvastatin was taken by some of our patients (30 out of $131 ; 22.9 \%$ ). However, as there was no difference in the proportion of patients taking simvastatin between the wild-type (30.6\%), single variant (35.4\%) and double variant groups (14.3\%), this would not have affected the generalisability of our findings.

Whilst there is substantial evidence associating CYP2C9 and VKORC1 variants with warfarin dose requirements $(5,12,20,21), C Y P 2 C 9$ is the more important gene determining initial anticoagulant control, with patients with mutant genes achieving a therapeutic INR faster than the wild-type ones (27). Many pharmacogenetics-based warfarin dosing algorithms have been developed for achieving a target INR of 2-3, the greatest potential for benefit for which is before initiation and early in the course of therapy (28-30). The GIFT randomized trial, the first of its kind powered to assess clinical outcomes, reported that use of a genotype-guided dosing algorithm for CYP2C9*2 and *3, CYP4F2*3 and VKORC1-1639 versus a clinical dosing algorithm in elective hip and knee arthroplasty patients treated with perioperative warfarin resulted in a $27 \%$ reduction in the combined risk of major bleeding, INR of 4 or greater, venous thromboembolism, or death (31).

Investigations of the cost-effectiveness of using pharmacogenetics-based information for warfarin therapy have historically considered initiation and continuation of warfarin, balancing the costs associated with genotyping against any reduction in adverse events over a fixed period, and have demonstrated inconsistent results. We set out in this study to investigate whether a pharmacogenetics-based algorithm incorporating CYP2C9 genotype and patient age could, in addition, be clinically useful for estimating the correct length of time needed for individual patients to stop taking warfarin prior to surgery, optimising the time patients spend off warfarin and thereby also potentially reducing the amount of interim 
anticoagulation use with low molecular weight heparin given for high-risk patients while their INR is sub-therapeutic (9). This would be beneficial as, although such bridging therapy is associated with low rates of inpatient thromboembolism in elective general surgical patients, it is also associated with bleeding complications (32). Our results, which demonstrate that CYP2C9 genotype does influence how quickly a patient withdrawing from warfarin will reach a safe INR before surgery indicate that including such genetic information into clinical practice has the potential to improve the cost-effectiveness of temporary warfarin withdrawal, although as age and CYP2C9 genotype explained only $16 \%$ of variability, we conclude that establishing the extent of the influence of other factors both known to affect an individual's response to warfarin such as disease states, diet and alcohol, and as yet unknown, would be required to improve the clinical applicability of this approach. Future analyses of cost-effectiveness of pharmacogenetics-based algorithms incorporating both pharmacogenetics-guided dosing outcomes, and temporary withdrawal outcomes for patients in terms of the balance between savings made through reductions in the number of cancelled surgical procedures and thrombotic and bleeding complications, and the cost of genotyping, could guide health service providers in their decision making as to whether such an approach should be funded and incorporated into clinical practice.

\section{Acknowledgements}

The authors acknowledge the contribution of research staff who were responsible for study co-ordination, patient recruitment and data handling; Lynne Robson, Philip Hope, Lester Rivett. The authors also wish to thank all patients who participated in the study.

The Research was supported/funded by the National Institute for Health Research Newcastle Biomedical Research Centre based at Newcastle Hospitals NHS Foundation Trust and Newcastle University. The views expressed are those of the author(s) and not necessarily those of the NHS, the NIHR or the Department of Health.

\section{Conflict of Interest}


The authors declare no competing financial interests. 


\section{References}

1. Rettie AE, Wienkers LC, Gonzalez FJ, et al. Impaired (S)-warfarin metabolism catalysed by the R144C allelic variant of CYP2C9. Pharmacogenetics and Genomics 1994; 4(1): 39-42.

2. Haining RL, Hunter AP, Veronese ME, et al. Allelic variants of human cytochrome P450 2C9: baculovirus-mediated expression, purification, structural characterization, substrate stereoselectivity, and prochiral selectivity of the wild-type and $1359 \mathrm{~L}$ mutant forms. Archives of Biochemistry and Biophysics 1996; 333(2): 447-58.

3. Sullivan-Klose TH, Ghanayem BI, Bell DA, et al. The role of the CFP2C9-Leu 359 allelic variant in the tolbutamide polymorphism. Pharmacogenetics and Genomics 1996; 6(4): 341-9.

4. Rost S, Fregin A, Ivaskevicius V, et al. Mutations in VKORC1 cause warfarin resistance and multiple coagulation factor deficiency type 2. Nature 2004; 427(6974): 537-41.

5. Sconce EA, Khan TI, Wynne HA, et al. The impact of CYP2C9 and VKORC1 genetic polymorphism and patient characteristics upon warfarin dose requirements: proposal for a new dosing regimen. Blood 2005; 106(7): 2329-33.

6. Rieder MJ, Reiner AP, Gage BF, et al. Effect of VKORC1 haplotypes on transcriptional regulation and warfarin dose. The New England journal of medicine 2005; 352(22): 2285-93.

7. Jorgensen AL, FitzGerald RJ, Oyee J, et al. Influence of CYP2C9 and VKORC1 on patient response to warfarin: a systematic review and meta-analysis. PloS one 2012; 7(8): e44064.

8. Hylek EM, Regan S, Go AS, et al. Clinical predictors of prolonged delay in return of the international normalized ratio to within the therapeutic range after excessive anticoagulation with warfarin. Ann Intern Med 2001; 135(6): 393-400.

9. Douketis JD, Spyropoulos AC, Spencer FA, et al. Perioperative management of antithrombotic therapy: Antithrombotic Therapy and Prevention of Thrombosis, 9th ed: American College of Chest Physicians Evidence-Based Clinical Practice Guidelines. Chest 2012; 141(2 Suppl): e326S-50S.

10. White RH, McKittrick T, Hutchinson R, et al. Temporary discontinuation of warfarin therapy: changes in the international normalized ratio. Ann Intern Med 1995; 122(1): 40-2.

11. Kovacs MJ, Kearon C, Rodger M, et al. Single-arm study of bridging therapy with lowmolecular-weight heparin for patients at risk of arterial embolism who require temporary interruption of warfarin. Circulation 2004; 110(12): 1658-63.

12. Klein TE, Altman RB, Eriksson N, et al. Estimation of the warfarin dose with clinical and pharmacogenetic data. The New England journal of medicine 2009; 360(8): 753-64.

13. Abohelaika S, Wynne H, Avery $P$, et al. Influence of CYP2C9 polymorphism on the fall in International Normalized Ratio in patients interrupting warfarin therapy before elective surgery. Journal of Thrombosis and Haemostasis 2015; 13(8): 1436-40.

14. UK Department of Health. UK Chief Medical Officers' Alcohol Guidelines Review Summary of the proposed new guidelines. Available at:

https://www.gov.uk/government/uploads/system/uploads/attachment data/file/489795/summary. pdf. Accessed [Access date: 14/10/2016.

15. Naidong W, Lee JW. Papers from the Analysis and Pharmaceutical Quality Section presented at the Seventh Annual Meeting of the American Association of Pharmaceutical

ScientistsDevelopment and validation of a high-performance liquid chromatographic method for the quantitation of warfarin enantiomers in human plasma. Journal of Pharmaceutical and Biomedical Analysis 1993; 11(9): 785-92.

16. Rowland M, Tozer TN. Multiple dose regimens. Clinical Pharmacokenetics, Concepts and Implications 3rd ed Baltimore, MD: Williams \& Wilkins 1995: 83-105.

17. Wynne $H$, Cope L, Kelly $P$, et al. The influence of age, liver size and enantiomer concentrations on warfarin requirements. British journal of clinical pharmacology 1995; 40(3): 203-7. 18. Kadian-Dodov DL, van der Zee SA, Scott SA, et al. Warfarin pharmacogenetics: a controlled dose-response study in healthy subjects. Vasc Med 2013; 18(5): 290-7. 
19. Chartrungsan A. Comparison of Temporary Interruption of Warfarin Therapy for 3 and 5 days before Surgery in Thailand: A Randomized Controlled Trial. Siriraj Medical Journal 2013; 65(3): 69-72. 20. Miners JO, Birkett DJ. Cytochrome P4502C9: an enzyme of major importance in human drug metabolism. British journal of clinical pharmacology 1998; 45(6): 525-38.

21. Hillman MA, Wilke RA, Caldwell MD, et al. Relative impact of covariates in prescribing warfarin according to CYP2C9 genotype. Pharmacogenetics and Genomics 2004; 14(8): 539-47.

22. Li T, Lange LA, Li X, et al. Polymorphisms in the VKORC1 gene are strongly associated with warfarin dosage requirements in patients receiving anticoagulation. Journal of medical genetics 2006; 43(9): 740-4.

23. Van Booven D, Marsh S, McLeod H, et al. Cytochrome P450 2C9-CYP2C9. Pharmacogenetics and Genomics 2010; 20(4): 277-81.

24. Daly AK, Rettie AE, Fowler DM, et al. Pharmacogenomics of CYP2C9: Functional and Clinical Considerations. J Pers Med 2017; 8(1).

25. Gage BF, Eby C, Johnson JA, et al. Use of pharmacogenetic and clinical factors to predict the therapeutic dose of warfarin. Clin Pharmacol Ther 2008; 84(3): 326-31.

26. Gage BF, Eby C, Milligan PE, et al. Use of pharmacogenetics and clinical factors to predict the maintenance dose of warfarin. Thromb Haemost 2004; 91(1): 87-94.

27. Jorgensen AL, Al-Zubiedi S, Zhang JE, et al. Genetic and environmental factors determining clinical outcomes and cost of warfarin therapy: a prospective study. Pharmacogenet Genomics 2009; 19(10): 800-12.

28. Lenzini $\mathrm{P}$, Wadelius $\mathrm{M}$, Kimmel S, et al. Integration of genetic, clinical, and INR data to refine warfarin dosing. Clin Pharmacol Ther 2010; 87(5): 572-8.

29. Kimmel SE. Warfarin pharmacogenomics: current best evidence. J Thromb Haemost 2015; 13 Suppl 1: S266-71.

30. Pirmohamed M, Burnside G, Eriksson N, et al. A randomized trial of genotype-guided dosing of warfarin. The New England journal of medicine 2013; 369(24): 2294-303.

31. Gage BF, Bass AR, Lin H, et al. Effect of Genotype-Guided Warfarin Dosing on Clinical Events and Anticoagulation Control Among Patients Undergoing Hip or Knee Arthroplasty: The GIFT Randomized Clinical Trial. JAMA 2017; 318(12): 1115-24.

32. Breen DT, Chavalertsakul N, Paul E, et al. Perioperative complications in patients on lowmolecular-weight heparin bridging therapy. ANZ J Surg 2016; 86(3): 167-72. 
Table 1: CYP2C9 and VKORC1 genotype frequency distribution in the study population

\begin{tabular}{lr}
\hline CYP2C9 polymorphism, $\mathrm{n}(\%)$ & $72(56.7)$ \\
${ }^{*} 1{ }^{* 1}$ & $33(26.0)$ \\
${ }^{*}{ }^{* 2}$ & $15(11.8)$ \\
${ }^{*} 1{ }^{* 3}$ & $6(4.7)$ \\
${ }^{*} 2{ }^{* 2}$ & $1(0.8)$ \\
${ }^{*} 2{ }^{* 3}$ & \\
\hline$V K O R C 1$ polymorphism, (\%) & $49(38.6)$ \\
$G G$ & $63(49.6)$ \\
$A G$ & $15(11.8)$ \\
$A A$ &
\end{tabular}

N.B. Genotyping data missing for 4 patients 
Table 2: Influence of CYP2C9 polymorphism on mean (SD) warfarin dose and clearance

\begin{tabular}{|c|c|c|c|c|}
\hline & \multirow{2}{*}{$\begin{array}{c}\text { Warfarin } \\
\text { weekly dose } \\
(\mathrm{mg})\end{array}$} & \multicolumn{3}{|c|}{ Warfarin clearance (L/hr) } \\
\hline & & $S$ & $\mathrm{R}$ & Racemic \\
\hline \multicolumn{5}{|c|}{ CYP2C9 polymorphism } \\
\hline$* 1 * 1$ & $40(16)$ & $0.20(0.22)$ & $0.07(0.07)$ & $0.11(0.09)$ \\
\hline${ }^{*} 1 * 2 /{ }^{*} 1 * 3$ & $29(14)$ & $0.09(0.04)$ & $0.05(0.04)$ & $0.07(0.03)$ \\
\hline$* 2 * 2 /{ }^{*} 2 * 3$ & $25(9)$ & $0.09(0.05)$ & $0.07(0.01)$ & $0.07(0.02)$ \\
\hline
\end{tabular}

N.B. Data based on 127 patients as genotyping information was missing for 4 patients. 


\section{Highlights:}

- The fall in INR following warfarin withdrawal is influenced by CYP2C9 genotype and age.

- VKORC1 genotype does not influence the rate of decline in INR following warfarin withdrawal.

- A pharmacogenetic-guided approach could improve safety of temporary warfarin withdrawal prior to surgery. 